\title{
Proposta de aproveitamento em resíduos de pau- rainha (Brosimum rubescens) descartados pelo setor madeireiro
}

\author{
Willian HAYASIDA ${ }^{1}$, Arlison Silva de SOUSA ${ }^{2}$, Maria da Paz LIMA³, \\ Claudete Catanhede do NASCIMENTO ${ }^{4}$, Antonio Gilberto FERREIRA ${ }^{5}$
}

\section{RESUMO}

A indústria da madeira do estado de Amazonas (Brasil) contribui com a produção de uma quantidade grande de resíduos. Este trabalho visa indicar o uso final para espécie florestal pau-rainha (Brosimum rubescens Taubert, Moraceae). Os resíduos descartados durante o processamento mecânico da madeira foram utilizados na confecção de artefatos como: instrumentos musicais e artigos decorativos gerando resíduos menores (serragens). Foram obtidos extratos das serragens do cerne e alburno por maceração com hexano e metanol. O teor extrativo no cerne foi 19,87\% e a porcentagem (com relação a serragem) de xantiletina (2,2-dimetilcromeno cumarina) obtida foi $2,35 \%$. Não foi detectada a cumarina nos extratos do alburno. A xantiletina é reportada pelas atividades antiplaquetária, antifúngica e herbicida e alguns derivados possuem atividade em linhagens de células leucêmicas. A proposta de uso final adequado dos resíduos de pau-rainha para confecção de artefatos tem uma grande importância social e a busca de metabólitos secundários é bastante promissora pois estes podem ser transformados em novos produtos.

PALAVRAS-ChaVE: Madeiras tropicais, Resíduos madeireiros, Metabólitos secundários, Xantiletina

\section{Proposal of profite in residues of "pau-rainha" (Brosimum rubescens) discarded for the lumber sector}

\section{ABSTRACT}

Timber industry of Amazonas state (Brazil) contribute with the production of great amount of residues. This paper aims to indicating end-uses for this forest species pau-rainha (Brosimum rubescens Taubert, Moraceae). The residues discharged during the mechanical processing in timber production were used as manufactured goods such as: musical instruments and decorative articles generating less wastes in sawmills. They were carried sawdust of the heartwood and sapwood and extraction by maceration with hexane and methanol. The heartwood extractive yield was $19.87 \%$ and content of xanthyletin (2,2-dimethylchromene coumarin) was $2.35 \%$ with basis dry mass. It was not detected the coumarin in extracts of sapwood. Xanthyletin is reported as antiplatelet, antifungal and herbicide and some its derivatives have a leukaemic cells lineage activities. The proposal of adequate end-uses of "pau-rainha" as manufacture-goods is a great social benefit and the search of secondary metabolites is quite promising it can be transformed into novel products.

KEY WORDS:

Tropical woods, Wood residues, Secondary metabolites, Xanthyletin

\footnotetext{
1 Instituto Nacional de Pesquisas da Amazônia, hayasida_willian@hotmail.com

2 Instituto Nacional de Pesquisas da Amazônia, mdapaz@inpa.gov.br

3 Instituto Nacional de Pesquisas da Amazônia, mdapaz@inpa.gov.br

${ }^{4}$ Instituto Nacional de Pesquisas da Amazônia, catanhed@inpa.gov.br

5 Universidade Federal de São Carlos, giba@dq.ufscar.br
} 


\section{INTRODUÇÃO}

Brosimum rubescens Taubert [sin Brosimum paraense Huber], família Moraceae, é uma espécie arbórea, de altura que varia de 30 a 50 m, com ampla distribuição na Amazônia. Essa espécie é conhecida como "palo de sangre" e "palo negro" no Peru, "muirapiranga" e "pau-rainha" no Brasil (BrazFilho et al., 1972). Sua madeira é indicada para móveis de luxo, vigamentos, escadas, tacos de assoalho, instrumentos musicais, faqueados decorativos, objetos de adorno, entre outros. O cerne dessa madeira possui densidade alta $(0,90 \mathrm{~g} /$ $\mathrm{cm}^{3}$ ) e apresenta coloração vermelho-brilhante (Nascimento, 2000; Silva, 2002).

Silva \& Silva (1991) avaliaram a quantidade aproximada dos constituintes macromoleculares estruturais e extrativos presentes no cerne de pau-rainha da Amazônia: celulose $(56,6 \%)$, hemicelulose $(25,2 \%)$, lignina $(33,0 \%)$, extrativos ou metabólitos secundários (22,23\%). Estudos químicos sobre os metabólitos secundários indicam que os extrativos do cerne contêm grande quantidade de xantiletina (1) além de outras cumarinas (Figura 1) em quantidades relativamente pequenas como luvangetina (2), brosiparina (3), 7-desmetilsuberosina (4) e brosiprenina (5) (Braz-Filho et al., 1971).

A xantiletina é uma piranocumarina reportada pela atividade antiplaquetária (Teng et al., 1992) e anticancerígena (Gljnatilau \& Kingston, 1994), apresenta potencial herbicida (Anaya et al., 2005) e mostrou inibição à fungos simbióticos de formiga cortadeira (Godoy et al., 2005). O interesse nesta cumarina é também para uso como intermediário na síntese de compostos biologicamente ativos, como relatado por Magiatis e colaboradores (1998) que na busca de drogas antitumorais em produtos naturais, sintetizaram derivados mais potentes contra linhagens de células de leucêmicas (L-1210). Os derivados de xantiletina obtidos por Kim et al., (2001) têm recebido considerável atenção devido suas propriedades citotóxicas e analgésicas, além de ativos contra a bactéria Helicobacter pylori, causadora de úlceras.

Considerando que no Amazonas grande quantidade de resíduos de pau-rainha é descartado pelo setor madeireiro, o presente trabalho visou a análise de tais materiais brutos para avaliar a possibilidade de aproveitamento dos seus constituintes químicos.

\section{MATERIAL E MÉTODOS}

\section{PROCEDIMENTOS EXPERIMENTAIS GERAIS}

Os espectros de RMN foram obtidos em $\mathrm{CDCl}_{3}$, em aparelho Bruker modelo DRX-400 ( ${ }^{1} \mathrm{H}: 400 \mathrm{MHz} ;{ }^{13} \mathrm{C}: 100$ $\mathrm{MHz})$.

Para os fracionamentos utilizou-se colunas cromatográficas em celulose microcristalina Avicel (Merck) e para avaliação

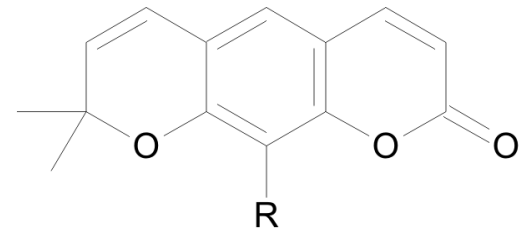

$1 \mathrm{R}=\mathrm{H}$

$2 \mathrm{R}=\mathrm{OMe}$

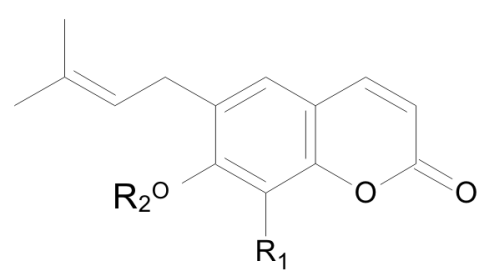

$3 \mathrm{R}_{1}=\mathrm{OH}, \mathrm{R}_{2}=\mathrm{Me}$

$4 \mathrm{R}_{2}=\mathrm{R}_{1}=\mathrm{H}$

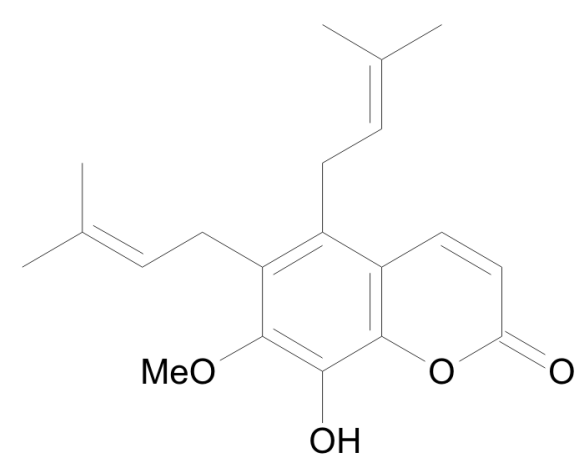

5

Figura 1 - Cumarinas identificadas no extrativo do cerne de pau-rainha

das frações e substâncias utilizou-se cromatografia em camada delgada comparativa em placas de sílica gel G60 $(0,20 \mathrm{~mm}$; Machery-Nagel).

\section{OBTENÇÃO E IDENTIFICAÇÃO DAS SERRAGENS}

A matéria-prima (resíduos) utilizada foi coletada durante o processamento mecânico da madeira certificada de pau-rainha, na empresa Mil Madeireira, localizada na AM 010 do KM 234 (Manaus-Itacoatiara), no âmbito do desenvolvimento do projeto PAPPE/FAPEAM e FINEP.

A identificação dos resíduos foi realizada na Xiloteca da Coordenação de Pesquisas em Produtos Florestais (CPPF) do 
INPA, por análises anatômicas e comparação com as amostras depositadas no acervo X-10075, X-10076 eX-10077. Durante a confecção dos artefatos como instrumentos musicais e artigos decorativos, foram gerados resíduos menores (serragens) do cerne e alburno, os quais foram selecionados para avaliação dos constituintes químicos fixos e/ou voláteis.

\section{EXTRAÇÕES DOS CONSTITUINTES QUIIMICOS}

Para obtenção dos constituintes químicos voláteis, 298 g de serragem do cerne foi submetida à extração por hidrodestilação em sistema do tipo Clevenger, durante 4 horas, acoplado a sistema de refrigeração. Quanto aos constituintes químicos fixos, preparou-se extratos brutos do cerne utilizando-se $100 \mathrm{~g}$ de serragem em maceração à frio com hexano seguida por metanol (sete dias em cada solvente) o qual após a evaporação dos solventes (evaporador rotativo), forneceu $269 \mathrm{mg}$ do extrato obtido em hexano e 19,6 g em metanol. Para monitorar a presença de xantiletina por cromatografia em camada delgada (CCD), utilizou-se uma amostra padrão previamente identificada. A recristalização (hexano: gotas de acetona) do extrato obtido em hexano, forneceu $174 \mathrm{mg}$ de xantiletina (1). O extrato obtido em metanol foi particionado em diclorometano (fase 1) e posteriormente em acetato de etila (fase 2). Avaliação em CCD mostrou a presença da xantiletina na fase 2. Para purificação da xantiletina, a fase 2 após concentrada foi submetida a filtração em coluna de celulose para remoção dos pigmentos (vermelho intenso) utilizando diclorometano como eluente, fornecendo material cristalino que após tratamento em éter etílico, obteve-se 2,18 $\mathrm{g}$ de xantiletina (1). Os extratos da serragem do alburno foram obtidos utilizando-se o mesmo procedimento do cerne, no entanto estes não apresentaram xantiletina.

\section{RESULTADOS E DISCUSSÃO}

A hidrodestilação das serragens (cerne e alburno) de Brosimum rubescens forneceu apenas traços de óleos essenciais, não sendo possível a coleta para a identificação dos seus componentes químicos.

Quanto aos constituintes químicos fixos, a xantiletina foi detectada como predominante nos extratos do cerne de paurainha. O padrão de xantiletina utilizado para comparação em CCD durante o fracionamento dos extratos foi previamente isolado de Spathelia excelsa (Rutaceae) e para confirmação da estrutura, foram efetuadas análises por RMN da substância 1, cujos dados foram comparados com os publicados para substância padrão (Tabela 1).

$\mathrm{O}$ espectro de $\mathrm{RMN}{ }^{1} \mathrm{H}$ apresenta sinais de hidrogênios olefínicos característicos de piranocumarinas em $\delta$ 6,34 $(\mathrm{H}-3)$ e $\delta 7,59(\mathrm{H}-4) ; \delta 5,70\left(\mathrm{H}-3^{\prime}\right)$ e $\delta 6,35$ (H-4’). Os deslocamentos químicos dos hidrogênios aromáticos em singletos em $\delta 7,05$ e $\delta 6,72$ indicaram a linearidade da cumarina. Os sinais referentes às metilas foram observados em $\delta 1,46$ integrando para 6 hidrogênios.

No espectro de RMN ${ }^{13} \mathrm{C}$ observou-se a presença de quatorze carbonos. $\mathrm{O}$ sinal da carbonila foi registrado em $\delta$ 161,24 e dos carbonos olefínicos em $\delta$ 131,21 (C-3) e $\delta 143,30 \quad(\mathrm{C}-4) ; \delta 104,39\left(\mathrm{C}^{\prime} 3^{\prime}\right)$ e $\delta 113,02\left(\mathrm{C}-4^{\prime}\right)$. Os deslocamentos químicos dos demais sinais foram similares aos obtidos para substância padrão (Lima 2000).

Tabela 1 - Deslocamentos químicos nos espectros de RMN ${ }^{1} \mathrm{H}$ e ${ }^{13} \mathrm{C}$ da substância 1

\begin{tabular}{|c|c|c|c|}
\hline Posição & $\begin{array}{c}\mathrm{RMN}{ }^{1} \mathrm{H}\left(\mathrm{CDCl}_{3}\right) \\
\text { experimental }\end{array}$ & $\begin{array}{c}\mathrm{RMN}{ }^{13} \mathrm{C}\left(\mathrm{CDCl}_{3}\right) \\
\text { experimental }\end{array}$ & $\begin{array}{c}\text { RMN }{ }^{13} \mathrm{C}\left(\mathrm{CDCl}_{3}\right) \\
\text { Literatura (Lima, 2000) }\end{array}$ \\
\hline 2 & --- & 161,15 & 161,24 \\
\hline 3 & $6,34(d ; 9,6 \mathrm{~Hz})$ & 131,21 & 131,24 \\
\hline 4 & $7,59(d ; 9,6 \mathrm{~Hz})$ & 143,30 & 143,36 \\
\hline 5 & $7,05(s)$ & 124,75 & 124,76 \\
\hline 6 & --- & 118,50 & 118,51 \\
\hline 7 & --- & 156,83 & 156,83 \\
\hline 8 & $6,72(s)$ & 120,77 & 120,76 \\
\hline 9 & --- & 155,55 & 155,43 \\
\hline 10 & --- & 112,71 & 112,71 \\
\hline ' & --- & 77,72 & 77,70 \\
\hline 3' & $5,70(d ; 8,8 \mathrm{~Hz})$ & 104,39 & 104,42 \\
\hline $4^{\prime}$ & $6,35(d ; 8,8 \mathrm{~Hz})$ & 113,02 & 113,03 \\
\hline Me & 1,46 & 28,33 & 28,34 \\
\hline-- & 1,46 & 28,33 & 28,13 \\
\hline
\end{tabular}

A madeira da espécie mencionada é utilizada pela empresa sediada no Amazonas para atender a demanda do mercado externo, pelas excelentes propriedades tecnológicas e coloração avermelhada do cerne. Por ser utilizada para exportação, gera resíduos em torno de 60 à $75 \%$ durante o processamento mecânico em razão da exigência do consumidor quando determina as dimensões e qualidade do produto. Geralmente os resíduos são utilizados pela empresa para geração de energia. No âmbito deste trabalho, resíduos do cerne foram utilizados em instrumentos musicais por possuir alta sonoridade e resistência mecânica. A preferência pelo cerne de pau-rainha para confecção de instrumentos musicais é devido a cor, resistência, fibra reta e a sonoridade produzida no teste de toque. Há indicativo de que a presença de cristais em madeira é um dos fatores para obtenção de um violão com melhor acústica, isso sugere que a abundância dos cristais de xantiletina contribui para a boa sonoridade dos instrumentos musicais.

O teor extrativo no cerne foi de $19,87 \%$ e a substância predominante obtida com percentual de $2,35 \%$ com relação à serragem e 11,82\% em relação aos extratos brutos. Assim, estima-se que numa tora de pau-rainha com $4,52 \mathrm{~m}$ de comprimento x 53,5 cm de diâmetro médio, retirando-se a 
casca de $1 \mathrm{~cm}$ e o alburno em torno de $13 \%$, cerca de $18 \mathrm{~kg}$ de xantiletina está presente no cerne.

As cumarinas constituem a principal classe O-heterocíclica em produtos naturais com largo espectro de atividade biológica. Apresentam ampla distribuição em plantas de angiospermas, no entanto as piranocumarinas têm ocorrência mais restrita na natureza. Na família Moraceae, a xantiletina é encontrada em Brosimum gaudichaudii (1999), B. rubescens (Braz-Filho et al., 1971; 1972) e Ficus beecheyana (Lee, et al., 2004), sendo a madeira de B. rubescens (pau-rainha) considerada uma fonte excelente desta cumarina promissora de atividades biológicas.

Nos extratos obtidos da serragem do alburno não foi detectada a presença de xantiletina e esta parte da madeira é susceptível ao ataque de fungos e insetos, o que nos permite sugerir que a resistência do cerne nestes organismos está relacionada com a presença desta cumarina.

No processamento mecânico utilizado pelo setor madeireiro são geradas grandes quantidades de resíduos de pau-rainha os quais são parcialmente aproveitados para gerar energia, comercialização de produtos de terceira qualidade ou doados para confecção de artefatos. Considerando que estes são resíduos de excelente qualidade, a proposta de confecção de pequenos objetos tem uma grande importância social e o reaproveitamento das serragens em busca de metabólitos secundários é bastante promissor pois podem ser transformados em bioprodutos (Esquema 1).

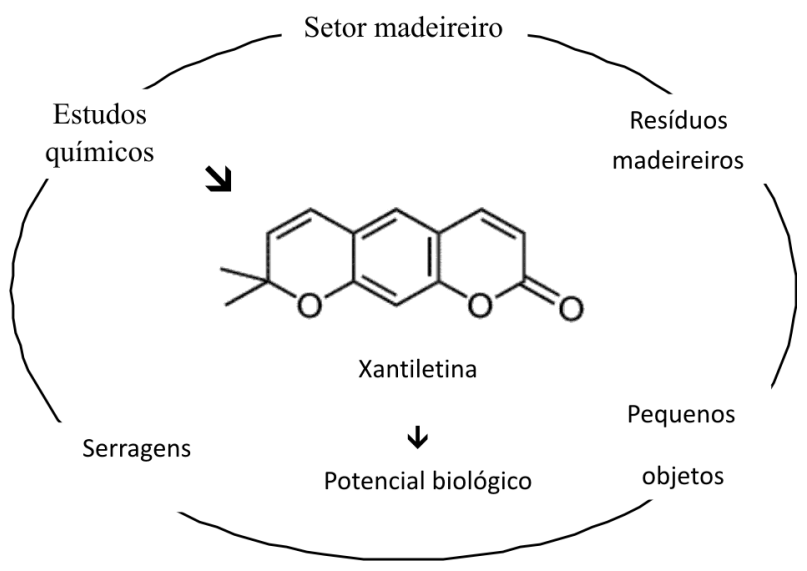

Esquema 1 - Cadeia de aproveitamento dos resíduos de Pau-rainha

\section{BIBLIOGRAFIA CITADA}

Anaya, A. L.; Rubalcava, M.M.; Ortega, R.C.; Santana, C.G.; Monterrubio, P.N.S.; Bautista, B.E.H.; Rachel, M.R. 2005. Allelochemicals from Stauranthus perforatus, a Rutaceous tree of the Yucatan Peninsula, Mexico. Phytochemistry, 66 (4): $487-$ 494.
Braz-Filho, R.; Magalhães, A.F.; Gottlieb, O.R. 1971. A química de Moraceae no Brasil-II: Brosiprenina and outras cumarinas de Brosimum rubescens. Anais da Academia Brasileira de Ciências, 43 (3-4): 585-586.

Braz-Filho, R.; Magalhães, A.F.; Gottlieb, R. 1972. Coumarins from Brosimum rubescens. Phytochemistry, 11(11): 3307-3310.

Gljnatilau, A.A.L.; Kingston, D.G.I. 1994. Biological activity of some coumarins from Sri Lankan Rutaceae. Journal of Natural Products, 57, (4): 518-520.

Godoy, M.F.P.; Victor, S.R.; Bellini, A.M.; Guerreiro, G.; Rocha W.C.; Bueno, O.C.; Hebling, M.J.A.; Baccci-Jr, M.; Silva, M.F.G.F.; Vieira, P.C.; Fernandes J.B.; Pagnocca F.C. 2005. Inhibition of the symbiotic fungus of leaf-cutting ants by coumarins. Journal of the Brazilian Chemical Society, 16(3B): 669-672.

Kim, S.; Ko, H.; Son, S.; Shin, K.J.; Kimb, D.J. 2001. Enantioselective syntheses of (+)-decursinol and (+)-trans-decursidinol. Tetrahedron Letters, 42: 7641-7643.

Lee, C.K.; Lu, C.K.; Kuo,_Y.H.; Chen, J.Z.; Sun, G.Z. 2004. New prenylated flavones from the roots of Ficus beecheyana. Journal of the Chinese Chemical Society, 51(2):437-441.

Lima, M.P. 2000. Investigação fitoquímica e quimiossistemática de Trattinickia burserifolia, Crepidospermum rhoifolium, Trattinickia rhoifolia, Dacryodes sp. (Burseraceae) e Spathelia excelsa (Rutaceae). Tese de Doutorado, Universidade Federal de São Carlos. São Carlos, São Paulo. 350pp.

Nascimento, C.C. 2000. Variabilidade e desenvolvimento de modelos para estimar propriedades da madeira de quatro espécies da Amazônia. Tese de Doutorado. Instituto Nacional de Pesquisas da Amazônia/Fundação Universidade do Amazonas, Manaus, Amazonas. 154pp.

Magiatis, P.; Melliou, E.; Skaltsounis, A.L.; Mitaku, S.; Léonce, S.; Renard, P.; Pierré, A.; Atassi, G. 1998. Synthesis and cytotoxic activity of pyranocoumarins of the seselin and xanthyletin series. Journal of Natural Products, 61 (8): 982-986.

Silva, A.C. 2002. Madeiras da Amazônia: características gerais, nome vulgar e usos. Manaus: Edição SEBRAE. 237pp.

Silva, A.C.; Silva, M.B.C. 1991. Influência da anatomia e química nas propriedades da madeira. Edição UTAM, 17p.

Teng, C.M.; Li, H.L.; Wu, T.S.; Huang, S.C.; Huang, T.F. 1992. Antiplatelet actions of some coumarin compounds isolated from plant sources. Thrombosis Research, 66 (5): 549-557.

Vieira, I.C.; Mathias, L.; Monteiro, V.F.F.; Braz-Filho, R.; Edson Rodrigues-Filho, E. 1999. A New Coumarin from Brosimum gaudichaudii Trecul. Natural Product Research, 13(1): 47-52.

Recebido em 28/12/2007

Aceito em 30/06/2008 